\title{
A PROCEDURE FOR GENERATING INFINITE SERIES IDENTITIES
}

\author{
ANTHONY A. RUFFA
}

Received 3 March 2004

\begin{abstract}
A procedure for generating infinite series identities makes use of the generalized method of exhaustion by analytically evaluating the inner series of the resulting double summation. Identities are generated involving both elementary and special functions. Infinite sums of special functions include those of the gamma and polygamma functions, the Hurwitz Zeta function, the polygamma function, the Gauss hypergeometric function, and the Lerch transcendent. The procedure can be automated with Mathematica (or equivalent software).
\end{abstract}

2000 Mathematics Subject Classification: 26A06, $26 B 15$.

1. Introduction. The generalized method of exhaustion [5] provides series expansions for Riemann integrals of the form

$$
\begin{aligned}
\int_{a}^{b} f(x) d x & =(b-a) \lim _{N \rightarrow \infty} \sum_{n=1}^{N} \sum_{m=1}^{2^{n}-1}(-1)^{m+1} 2^{-n} f\left(a+\frac{m(b-a)}{2^{n}}\right) \\
& =(b-a) \sum_{n=1}^{\infty} \sum_{m=1}^{2^{n}-1}(-1)^{m+1} 2^{-n} f\left(a+\frac{m(b-a)}{2^{n}}\right) .
\end{aligned}
$$

The first expression is identical to the limit of the Riemann sum of order $2^{N}$ for $f(x)$ over $[a, b]$, except for the missing endpoints $f(a)$ or $f(b)$, which can be neglected when $N \rightarrow \infty$. Thus, convergence of the expansion is guaranteed for $N \rightarrow \infty$ when the integral exists.

The double summation expression can be reduced to that of a single summation when the inner finite series can be evaluated analytically. Analytical sums are occasionally expressible in terms of elementary functions or (more often) in terms of special functions. Some examples of special functions are the gamma and polygamma functions, the Gauss hypergeometric function, the Hurwitz Zeta function, and the Lerch transcendent. Identities involving these and other functions can stimulate solutions to engineering or physics problems [6], or lead to new insights into existing solutions.

The analytical evaluation of the inner series can often be automated with the use of software such as Mathematica. A few of the more important results are presented in this paper, along with a table of integrals permitting analytical evaluations of the inner series.

2. Generation of identities. The simplest analytic expressions resulting from the use of (1.1) involve geometric series, one form of which is as follows:

$$
\sum_{m=1}^{2^{n}-1}(-1)^{m+1} e^{m x / 2^{n}}=1+\frac{e^{x}-1}{e^{x / 2^{n}}+1} .
$$


This result can then be applied to the following definite integral:

$$
\begin{aligned}
\int_{0}^{x} e^{z} d z & =e^{x}-1=x \sum_{n=1}^{\infty} \sum_{m=1}^{2^{n}-1}(-1)^{m+1} 2^{-n} e^{m x / 2^{n}} \\
& =x \sum_{n=1}^{\infty} 2^{-n}\left(1+\frac{e^{x}-1}{e^{x / 2^{n}}+1}\right) \\
& =x+\left(e^{x}-1\right) x \sum_{n=1}^{\infty} \frac{2^{-n}}{e^{x / 2^{n}}+1}
\end{aligned}
$$

or

$$
\frac{1}{x}=\frac{1}{e^{x}-1}+\sum_{n=1}^{\infty} \frac{2^{-n}}{e^{x / 2^{n}}+1}
$$

Integration (and some algebra) leads to

$$
\ln x=(x-1) \prod_{n=1}^{\infty} \frac{2}{1+x^{2^{-n}}}
$$

Setting $x=i \theta$ in (2.2) and following a similar procedure leads to the well-known result [4]

$$
\sin \theta=\theta \prod_{n=1}^{\infty} \cos \left(\frac{\theta}{2^{n}}\right)
$$

A second expression for the logarithm can be derived from (1.1) as follows:

$$
\int_{1}^{x} \frac{d z}{z}=\ln x=\sum_{n=1}^{\infty} \sum_{m=1}^{2^{n}-1} \frac{(-1)^{m+1}(x-1)}{2^{n}+m(x-1)} .
$$

Using the identity [1]

$$
\psi_{0}(z)=\int_{0}^{\infty}\left[\frac{e^{-t}}{t}-\frac{e^{-z t}}{1-e^{-t}}\right] d t
$$

where $\psi_{0}(x)=(d / d x) \ln \Gamma(x)$ is the digamma function, it can be shown that

$$
\begin{gathered}
\psi_{0}\left(2^{n}+\frac{2^{n}}{(x-1)}\right)-\psi_{0}\left(1+\frac{2^{n}}{(x-1)}\right)=\sum_{m=1}^{2^{n}-1} \frac{(x-1)}{2^{n}+m(x-1)}, \\
\psi_{0}\left(2^{n}+\frac{1}{2}+\frac{2^{n}}{(x-1)}\right)-\psi_{0}\left(\frac{1}{2}+\frac{2^{n}}{(x-1)}\right)=\sum_{m=1}^{2^{n}} \frac{2(x-1)}{2^{n+1}+(2 m-1)(x-1)},
\end{gathered}
$$


and thus,

$$
\begin{aligned}
\sum_{m=1}^{2^{n}-1} \frac{(-1)^{m+1}(x-1)}{2^{n}+m(x-1)}= & \frac{1}{2} \psi_{0}\left(1+\frac{2^{n-1}}{x-1}\right)-\frac{1}{2} \psi_{0}\left(\frac{1}{2}+\frac{2^{n-1}}{x-1}\right) \\
& +\frac{1}{2} \psi_{0}\left(\frac{1}{2}+\frac{2^{n-1} x}{x-1}\right)-\frac{1}{2} \psi_{0}\left(\frac{2^{n-1} x}{x-1}\right)
\end{aligned}
$$

This leads to the result

$$
\begin{aligned}
\ln x= & \frac{1}{2} \sum_{n=0}^{\infty}\left[\psi_{0}\left(1+\frac{2^{n}}{x-1}\right)-\psi_{0}\left(\frac{1}{2}+\frac{2^{n}}{x-1}\right)\right] \\
& +\frac{1}{2} \sum_{n=0}^{\infty}\left[\psi_{0}\left(\frac{1}{2}+\frac{2^{n} x}{x-1}\right)-\psi_{0}\left(\frac{2^{n} x}{x-1}\right)\right] .
\end{aligned}
$$

The next example involves the exponential integral, that is,

$$
\int_{0}^{x} \frac{e^{z}-1}{z} d z=\operatorname{Ei}(x)-\ln x-\gamma=\sum_{n=1}^{\infty} \sum_{m=1}^{2^{n}-1}(-1)^{m+1} \frac{e^{m x / 2^{n}}-1}{m} .
$$

Here, $\gamma$ is the Euler-Mascheroni constant. From (2.13), Mathematica yields the result

$$
\begin{aligned}
\sum_{m=1}^{2^{n}-1}(-1)^{m+1} \frac{e^{m x / 2^{n}}-1}{m}= & \ln \left(\frac{1}{2}+\frac{1}{2} e^{x / 2^{n}}\right)+2^{-n} e^{x}{ }_{2} F_{1}\left(2^{n}, 1,1+2^{n},-e^{x / 2^{n}}\right) \\
& +\frac{1}{2} \psi_{0}\left(2^{n-1}\right)-\frac{1}{2} \psi_{0}\left(\frac{1}{2}+2^{n-1}\right)
\end{aligned}
$$

where ${ }_{2} F_{1}$ is the Gauss hypergeometric function. Noting that $[2,3,7]$

$$
\gamma=\frac{1}{2} \sum_{n=1}^{\infty}\left[\psi_{0}\left(\frac{1}{2}+2^{n-1}\right)-\psi_{0}\left(2^{n-1}\right)\right]=\sum_{n=1}^{\infty} \sum_{k=0}^{\infty} \frac{(-1)^{k}}{2^{n}+k}
$$

an identity for the exponential integral becomes

$$
\operatorname{Ei}(x)=\ln x+\sum_{n=1}^{\infty} \ln \left(\frac{1}{2}+\frac{1}{2} e^{x / 2^{n}}\right)+e^{x} \sum_{n=1}^{\infty} 2^{-n}{ }_{2} F_{1}\left(2^{n}, 1,1+2^{n},-e^{x / 2^{n}}\right) .
$$

The identity (2.6) leads to

$$
\sum_{n=1}^{\infty} \ln \left(\frac{1}{2}+\frac{1}{2} e^{x / 2^{n}}\right)=\ln \left(e^{x}-1\right)-\ln x
$$


TABLE 2.1. Some integrals having summable inner series.

\begin{tabular}{cc}
\hline Integral & Infinite series of the following function(s) \\
\hline $\int_{0}^{x}(\sin z / z) d z$ & Gauss hypergeometric function \\
$\int_{0}^{x}\left(\sin ^{2} z / z^{2}\right) d z$ & Lerch transcendent, trigamma function \\
$\int_{0}^{x}\left(\sin ^{3} z / z^{3}\right) d z$ & Lerch transcendent \\
$\int_{0}^{x}(\sin z / \sqrt{z}) d z$ & Lerch transcendent \\
$\int_{0}^{x} \ln (a+b z) d z$ & Logarithm of gamma function \\
$\int_{0}^{x}[\ln (a+b z)]^{2} d z$ & Logarithm of gamma function, Hurwitz Zeta function \\
$\int_{0}^{x}\left(d z /\left(a+z^{2}\right)\right)$ & Digamma function, hyperbolic tangent \\
$\int_{0}^{x}(z d z /(a+z))$ & Digamma function \\
$\int_{0}^{x}\left(\sin ^{2} z /\left(a+z^{2}\right)\right) d z$ & Gauss hypergeometric function, digamma function \\
$\int_{0}^{x}(a+b z)^{c} d z$ & Hurwitz Zeta function \\
$\int_{0}^{x} e^{z} \ln z d z$ & Lerch transcendent \\
\hline
\end{tabular}

so that

$$
\operatorname{Ei}(x)=\ln \left(e^{x}-1\right)+e^{x} \sum_{n=1}^{\infty} 2^{-n} F_{1}\left(2^{n}, 1,1+2^{n},-e^{x / 2^{n}}\right) .
$$

An identity for the gamma function can be generated with the integral

$$
\int_{0}^{x} z^{a} e^{-z} d z=\Gamma(a+1)-\Gamma(a+1, x)=x \sum_{n=1}^{\infty} \sum_{m=1}^{2^{n}-1}(-1)^{m+1} 2^{-n}\left(\frac{m x}{2^{n}}\right)^{a} e^{-m x / 2^{n}}
$$

It can be shown that

$$
\begin{aligned}
& \sum_{m=1}^{2^{n}-1}(-1)^{m+1} 2^{-n}\left(\frac{m x}{2^{n}}\right)^{a} e^{-m x / 2^{n}} \\
& \quad=2^{-n(1+a)} x^{a}\left[e^{-x} \Phi\left(-e^{-x / 2^{n}},-a, 2^{n}\right)+e^{-x / 2^{n}} \Phi\left(-e^{-x / 2^{n}},-a, 1\right)\right] .
\end{aligned}
$$

Here, $\Phi(z, s, b)=\sum_{k=0}^{\infty} z^{k}(b+k)^{-s}$ is the Lerch transcendent. The identity for the gamma function is as follows:

$$
\begin{aligned}
& \Gamma(a+1)-\Gamma(a+1, x) \\
& \quad=x^{a+1} \sum_{n=1}^{\infty} 2^{-n(1+a)}\left[e^{-x} \Phi\left(-e^{-x / 2^{n}},-a, 2^{n}\right)+e^{-x / 2^{n}} \Phi\left(-e^{-x / 2^{n}},-a, 1\right)\right] .
\end{aligned}
$$

Table 2.1 lists some integrals permitting analytical evaluation of the inner series when expanded with (1.1), and the form of the resulting infinite series. 
3. Method of exhaustion variants. By using an approach similar to that in [5], a family of expansions similar to (1.1) can be generated for the definite integral, that is,

$$
\begin{aligned}
\int_{a}^{b} f(x) d x= & (b-a) \sum_{n=1}^{\infty} \sum_{m=1}^{3^{n}-1} 3^{-n} f\left(a+\frac{m(b-a)}{3^{n}}\right) \\
& -(b-a) \sum_{n=1}^{\infty} \sum_{m=1}^{3^{n-1}-1} 3^{-n+1} f\left(a+\frac{m(b-a)}{3^{n-1}}\right) \\
= & (b-a) \sum_{n=1}^{\infty} \sum_{m=1}^{4^{n}-1} 4^{-n} f\left(a+\frac{m(b-a)}{4^{n}}\right) \\
& -(b-a) \sum_{n=1}^{\infty} \sum_{m=1}^{4^{n-1}-1} 4^{-n+1} f\left(a+\frac{m(b-a)}{4^{n-1}}\right) \\
= & (b-a) \sum_{n=1}^{\infty} \sum_{m=1}^{5^{n}-1} 5^{-n} f\left(a+\frac{m(b-a)}{5^{n}}\right) \\
& -(b-a) \sum_{n=1}^{\infty} \sum_{m=1}^{5^{n-1}-1} 5^{-n+1} f\left(a+\frac{m(b-a)}{5^{n-1}}\right),
\end{aligned}
$$

and so forth.

Or, in general,

$$
\begin{aligned}
\int_{a}^{b} f(x) d x= & (b-a) \sum_{n=1}^{\infty} \sum_{m=1}^{p^{n}-1} p^{-n} f\left(a+\frac{m(b-a)}{p^{n}}\right) \\
& -(b-a) \sum_{n=1}^{\infty} \sum_{m=1}^{p^{n-1}-1} p^{-n+1} f\left(a+\frac{m(b-a)}{p^{n-1}}\right), \quad p=2,3,4, \ldots
\end{aligned}
$$

Note that

$$
\begin{aligned}
& (b-a) \sum_{n=1}^{N} \sum_{m=1}^{p^{n}-1} p^{-n} f\left(a+\frac{m(b-a)}{p^{n}}\right) \\
& \quad-(b-a) \sum_{n=1}^{N} \sum_{m=1}^{p^{n-1}-1} p^{-n+1} f\left(a+\frac{m(b-a)}{p^{n-1}}\right), \quad p=2,3,4, \ldots,
\end{aligned}
$$

is precisely the Riemann sum of order $p^{N}$ for $f(x)$ over $[a, b]$, except for the missing terms $f(a)$ or $f(b)$, which can be neglected in the limit $N \rightarrow \infty$. Thus, as $N \rightarrow \infty,(3.2)$ is valid for all Riemann integrable functions.

Each of these expressions leads to distinct identities, once the inner finite series is summed. A family of identities can thus be generated similar to each presented in the last section. For example, a family of identities for the logarithm similar to (2.6) is as 
follows:

$$
\begin{aligned}
\ln x & =(x-1) \prod_{n=1}^{\infty} \frac{2}{1+x^{1 / 2^{n}}} \\
& =(x-1) \prod_{n=1}^{\infty} \frac{3}{1+x^{1 / 3^{n}}+x^{2 / 3^{n}}} \\
& =(x-1) \prod_{n=1}^{\infty} \frac{4}{1+x^{1 / 4^{n}}+x^{2 / 4^{n}}+x^{3 / 4^{n}}} \\
& =(x-1) \prod_{n=1}^{\infty} \frac{5}{1+x^{1 / 5^{n}}+x^{2 / 5^{n}}+x^{3 / 5^{n}}+x^{4 / 5^{n}}} \\
& =(x-1) \prod_{n=1}^{\infty} \frac{p}{\sum_{q=0}^{p-1} x^{q / p^{n}}}, \quad p=2,3,4, \ldots
\end{aligned}
$$

A family of identities similar to (2.7) can also be generated

$$
\begin{aligned}
\sin x & =x \prod_{n=1}^{\infty} \frac{1}{2}\left(e^{-i x / 2^{n}}+e^{i x / 2^{n}}\right)=x \prod_{n=1}^{\infty} \cos \left(\frac{x}{2^{n}}\right) \\
& =x \prod_{n=1}^{\infty} \frac{1}{3}\left(e^{-2 i x / 3^{n}}+1+e^{2 i x / 3^{n}}\right)=x \prod_{n=1}^{\infty}\left(1-\frac{4}{3} \sin ^{2}\left(\frac{x}{3^{n}}\right)\right) \\
& =x \prod_{n=1}^{\infty} \frac{1}{4}\left(e^{-3 i x / 4^{n}}+e^{-i x / 4^{n}}+e^{i x / 4^{n}}+e^{3 i x / 4^{n}}\right) \\
& =x \prod_{n=1}^{\infty} \frac{1}{5}\left(e^{-4 i x / 5^{n}}+e^{-2 i x / 5^{n}}+1+e^{2 i x / 5^{n}}+e^{4 i x / 5^{n}}\right) \\
& =x \prod_{n=1}^{\infty} \sum_{q=1}^{p} \frac{1}{p} e^{(2 q-p-1) i x / p^{n}}, \quad p=2,3,4, \ldots .
\end{aligned}
$$

The results (3.4) and (3.5) are proven below. Consider the expression

$$
\begin{aligned}
\int_{0}^{x} e^{t} d t & =e^{x}-1 \\
& =x \sum_{n=1}^{\infty} \sum_{m=1}^{p^{n}-1} p^{-n} e^{m x / p^{n}}-x \sum_{n=1}^{\infty} \sum_{m=1}^{p^{n-1}-1} p^{-n+1} e^{m x / p^{n-1}}, \quad p=2,3,4, \ldots
\end{aligned}
$$

Each inner summation is a geometric series, leading to the result

$$
\sum_{m=1}^{p^{n}-1} p^{-n} e^{m x / p^{n}}-\sum_{m=1}^{p^{n-1}-1} p^{-n+1} e^{m x / p^{n-1}}=\frac{e^{x}-e^{x / p^{n}}}{p^{n}\left(e^{x / p^{n}}-1\right)}-\frac{e^{x}-e^{x / p^{n-1}}}{p^{n-1}\left(e^{x / p^{n-1}}-1\right)} .
$$


Next, making use of the identities

$$
\begin{aligned}
\left(e^{x / p^{n-1}}-1\right)= & \left(e^{x / p^{n}}-1\right)\left(\sum_{q=0}^{p-1} e^{q x / p^{n}}\right) \\
\left(e^{x / p^{n-1}}-1\right)\left(e^{x / p^{n}}-1\right) & =e^{(p+1) x / p^{n}}-e^{x / p^{n-1}}-e^{x / p^{n}}+1 \\
& =\left(e^{x / p^{n}}-1\right)^{2}\left(\sum_{q=0}^{p-1} e^{q x / p^{n}}\right),
\end{aligned}
$$

it can be shown that

$$
\begin{aligned}
\int_{0}^{x} e^{t} d t= & e^{x}-1 \\
= & x \sum_{n=1}^{\infty} \frac{e^{x}\left[e^{x / p^{n-1}}-p e^{x / p^{n}}+(p-1)\right]}{p^{n}\left(e^{x / p^{n}}-1\right)^{2}\left(\sum_{q=0}^{p-1} e^{q x / p^{n}}\right)} \\
& +x \sum_{n=1}^{\infty} \frac{(p-1) e^{(p+1) x / p^{n}}-p e^{x / p^{n-1}}+e^{x / p^{n}}}{p^{n}\left(e^{x / p^{n}}-1\right)^{2}\left(\sum_{q=0}^{p-1} e^{q x / p^{n}}\right)} .
\end{aligned}
$$

Noting that

$$
\left(e^{x / p^{n}}-1\right)^{2} \sum_{q=0}^{p-1} q e^{q x / p^{n}}=e^{x / p^{n}}-p e^{p x / p^{n}}+(p-1) e^{(p+1) x / p^{n}},
$$

we again show that

$$
\begin{aligned}
& e^{x / p^{n-1}}-p e^{x / p^{n}}+(p-1) \\
& =-\left(e^{x / p^{n}}-1\right)^{2} \sum_{q=0}^{p-1} q e^{q x / p^{n}}+(p-1)\left(e^{(p+1) x / p^{n}}-e^{x / p^{n-1}}-e^{x / p^{n}}+1\right) \\
& \quad=(p-1)\left(e^{x / p^{n}}-1\right)^{2}\left(\sum_{q=0}^{p-1} e^{q x / p^{n}}\right)-\left(e^{x / p^{n}}-1\right)^{2} \sum_{q=0}^{p-1} q e^{q x / p^{n}},
\end{aligned}
$$

so that (3.9) becomes

$$
e^{x}-1=x e^{x} \sum_{n=1}^{\infty} \frac{p-1}{p^{n}}-x\left(e^{x}-1\right) \sum_{n=1}^{\infty} \frac{\left(e^{x / p^{n}}-1\right)^{2} \sum_{q=0}^{p-1} q e^{q x / p^{n}}}{p^{n}\left(e^{x / p^{n}}-1\right)^{2} \sum_{q=0}^{p-1} e^{q x / p^{n}}}
$$

or

$$
\frac{1}{x}=\frac{e^{x}}{e^{x}-1}-\sum_{n=1}^{\infty} \frac{\sum_{q=1}^{p-1} q e^{q x / p^{n}}}{p^{n} \sum_{q=0}^{p-1} e^{q x / p^{n}}}
$$

Integrating leads to

$$
\ln x=\ln \left(e^{x}-1\right)-\sum_{n=1}^{\infty} \ln \left[\sum_{q=0}^{p-1} \frac{e^{q x / p^{n}}}{p}\right]
$$


or

$$
\frac{\left(e^{x}-1\right)}{x}=\prod_{n=1}^{\infty} \sum_{q=0}^{p-1} \frac{e^{q x / p^{n}}}{p} .
$$

When $x=\ln z$,

$$
\ln z=(z-1) \prod_{n=1}^{\infty}\left[\frac{p}{\sum_{q=0}^{p-1} z^{q / p^{n}}}\right], \quad p=2,3,4, \ldots
$$

The identities given by (3.5) can be proven if $x=i z$ in (3.13),

$$
\frac{1}{i z}=\frac{e^{i z}}{e^{i z}-1}-\sum_{n=1}^{\infty} \frac{\sum_{q=1}^{p-1} q e^{i q z / p^{n}}}{p^{n} \sum_{q=0}^{p-1} e^{i q z / p^{n}}}
$$

By evaluating the double geometric series, it can be shown that

$$
\begin{gathered}
\sum_{q=0}^{p-1} \sum_{r=0}^{p-1} e^{i(q-r) z / p^{n}}=\left[\sum_{q=1}^{p} e^{i(2 q-p-1) z / 2 p^{n}}\right]^{2}, \\
\operatorname{Im}\left\{\sum_{q=1}^{p-1} \sum_{r=0}^{p-1} q e^{i(q-r) z / p^{n}}\right\}=\operatorname{Im}\left\{\left[\sum_{q=1}^{p} e^{i(2 q-p-1) z / 2 p^{n}}\right] \sum_{q=1}^{p}(q-p / 2-1 / 2) e^{i(2 q-p-1) z / 2 p^{n}}\right\} .
\end{gathered}
$$

Taking the imaginary part of (3.17) and using (3.18) leads to

$$
-\frac{1}{z}=\frac{-\sin z}{2-2 \cos z}+\operatorname{Im}\left\{\sum_{n=1}^{\infty} \frac{\sum_{q=1}^{p}(q-p / 2-1 / 2) e^{i(2 q-p-1) z / 2 p^{n}}}{p^{n} \sum_{q=1}^{p} e^{i(2 q-p-1) z / 2 p^{n}}}\right\} .
$$

Integrating leads to

$$
-\ln z=-\ln \left[\sin \left(\frac{z}{2}\right)\right]+\sum_{n=1}^{\infty} \ln \left[\sum_{q=1}^{p} e^{i(2 q-p-1) z / 2 p^{n}}\right]+C
$$

or

$$
\sin \left(\frac{z}{2}\right)=z e^{C} \prod_{n=1}^{\infty}\left[\sum_{q=1}^{p} e^{i(2 q-p-1) z / 2 p^{n}}\right]
$$

Letting $y=z / 2$,

$$
\sin y=2 y e^{C} \prod_{n=1}^{\infty}\left[\sum_{q=1}^{p} e^{i(2 q-p-1) y / p^{n}}\right] .
$$


Finally, the integration constant $C$ can be evaluated by setting $y=0$, leading to

$$
\sin y=y \prod_{n=1}^{\infty}\left[\sum_{q=1}^{p} \frac{1}{p} e^{i(2 q-p-1) y / p^{n}}\right] .
$$

The remaining identities presented in the previous section also appear in other forms below. Many of the results lead to limit representations. For example, following a procedure similar to that leading to (2.12) leads to

$$
\begin{aligned}
\ln x= & \sum_{n=1}^{\infty}\left[\psi_{0}\left(\frac{p^{n} x}{x-1}\right)-\psi_{0}\left(1+\frac{p^{n}}{x-1}\right)\right] \\
& +\sum_{n=0}^{\infty}\left[\psi_{0}\left(1+\frac{p^{n}}{x-1}\right)-\psi_{0}\left(\frac{p^{n} x}{x-1}\right)\right], \quad p=2,3,4, \ldots
\end{aligned}
$$

or

$$
\ln x=\lim _{n \rightarrow \infty}\left[\psi_{0}\left(\frac{p^{n} x}{x-1}\right)-\psi_{0}\left(1+\frac{p^{n}}{x-1}\right)\right], \quad p=2,3,4, \ldots
$$

Setting $q=p^{n}$ leads to

$$
\ln x=\lim _{q \rightarrow \infty}\left[\psi_{0}\left(\frac{q x}{x-1}\right)-\psi_{0}\left(1+\frac{q}{x-1}\right)\right] .
$$

A procedure similar to that leading to (2.18) generates the following:

$$
\begin{aligned}
\operatorname{Ei}(x)-\ln x-\gamma= & e^{x} \sum_{n=1}^{\infty} p^{-n+1}{ }_{2} F_{1}\left(1, p^{n-1}, 1+p^{n-1}, e^{x / p^{n-1}}\right) \\
& -e^{x} \sum_{n=1}^{\infty} p^{-n}{ }_{2} F_{1}\left(1, p^{n}, 1+p^{n}, e^{x / p^{n}}\right) \\
& +\sum_{n=1}^{\infty} \ln \left(\frac{1-e^{x / p^{n-1}}}{1-e^{x / p^{n}}}\right)+\sum_{n=1}^{\infty}\left[\psi_{0}\left(p^{n-1}\right)-\psi_{0}\left(p^{n}\right)\right], \quad p=2,3,4, \ldots,
\end{aligned}
$$

or

$$
\begin{aligned}
& \operatorname{Ei}(x)-\ln x-\gamma=\ln \left(1-e^{x}\right)+e^{x}{ }_{2} F_{1}\left(1,1,2, e^{x}\right)+\psi_{0}(1) \\
& -e^{x} \lim _{n \rightarrow \infty} p^{-n}{ }_{2} F_{1}\left(1, p^{n}, 1+p^{n}, e^{x / p^{n}}\right) \\
& -\lim _{n \rightarrow \infty}\left[\ln \left(1-e^{x / p^{n}}\right)+\psi_{0}\left(p^{n}\right)\right], \quad p=2,3,4, \ldots
\end{aligned}
$$

Noting that [1] $\psi_{0}(1)=-\gamma$ and ${ }_{2} F_{1}\left(1,1,2, e^{x}\right)=-e^{-x} \ln \left(1-e^{x}\right)$, and setting $q=p^{n}$ leads to

$$
\operatorname{Ei}(x)=\ln x-\lim _{q \rightarrow \infty}\left[\psi_{0}(q)+\frac{e^{x}}{q}{ }_{2} F_{1}\left(1, q, 1+q, e^{x / q}\right)+\ln \left(1-e^{x / q}\right)\right] .
$$


Finally, a procedure similar to that leading to (2.21) generates the following:

$$
\begin{aligned}
& \Gamma(a+1)-\Gamma(a+1, x) \\
& \quad=-x^{1+a} \lim _{n \rightarrow \infty} p^{-n(1+a)}\left[e^{-x} \Phi\left(e^{-x / p^{n}},-a, p^{n}\right)-e^{-x / p^{n}} \Phi\left(e^{-x / p^{n}},-a, 1\right)\right] .
\end{aligned}
$$

Setting $q=p^{n}$ leads to

$$
\begin{aligned}
& \Gamma(a+1)-\Gamma(a+1, x) \\
& \quad=-x^{1+a} \lim _{q \rightarrow \infty} q^{-(1+a)}\left[e^{-x} \Phi\left(e^{-x / q},-a, q\right)-e^{-x / q} \Phi\left(e^{-x / q},-a, 1\right)\right] .
\end{aligned}
$$

While (1.1) leads to expressions in the form of infinite series, (3.2) often leads to limit representations, unless further operations (e.g., integration) are first applied to the two individual series expressions.

4. Summary. The examples presented here illustrate a procedure for generating infinite series identities, consisting of evaluating an integral using the generalized method of exhaustion, and then analytically summing the inner finite series. Mathematica (or equivalent software) can automate the second step, allowing rapid investigation of integrals.

\section{REFERENCES}

[1] M. Abromowitz and I. A. Stegun, Handbook of Mathematical Functions, US Government Printing Office, District of Columbia, 1972.

[2] D. H. Bailey and R. E. Crandall, Random generators and normal numbers, Experiment. Math. 11 (2002), no. 4, 527-546 (2003).

[3] M. Beeler, R.W. Gosper, and R. Schroeppel, Hakmem. AI Memo 239, Item 120, MIT, 1972, http://www.inwap.com/pdp10/hbaker/hakmem/series.html\#item120.

[4] I. S. Gradshteyn and I. M. Ryzhik, Table of Integrals, Series, and Products, Academic Press, New York, 1980.

[5] A. A. Ruffa, The generalized method of exhaustion, Int. J. Math. Math. Sci. 31 (2002), no. 6, 345-351.

[6] - The group velocity of electromagnetic waves diffracted by a small aperture, Wave Motion 35 (2002), no. 2, 157-161.

[7] T.-C. Wu, S.-H. Leu, S.-T. Tu, and H. M. Srivastava, A certain class of infinite sums associated with digamma functions, Appl. Math. Comput. 105 (1999), no. 1, 1-9.

Anthony A. Ruffa: Naval Undersea Warfare Center Division, 1176 Howell Street, Newport, RI 02841, USA

E-mail address: ruffaaa@npt. nuwc . navy.mi 1 


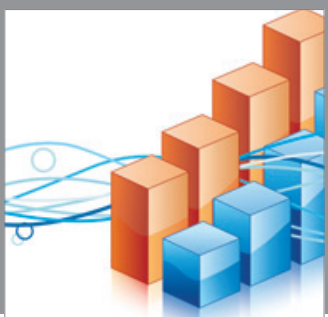

Advances in

Operations Research

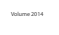

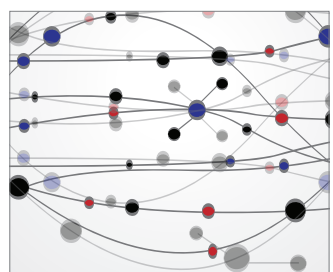

\section{The Scientific} World Journal
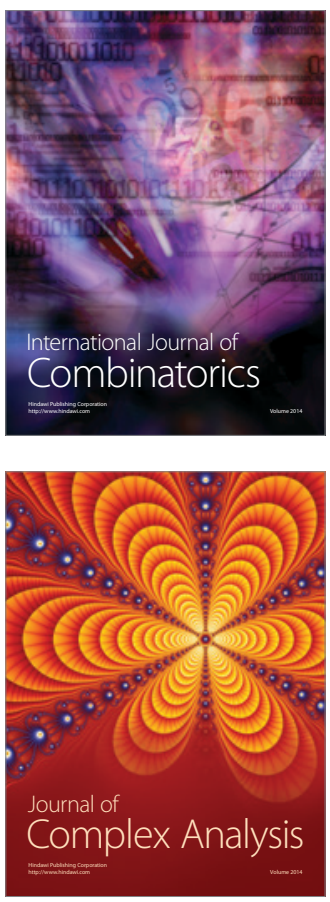

International Journal of

Mathematics and

Mathematical

Sciences
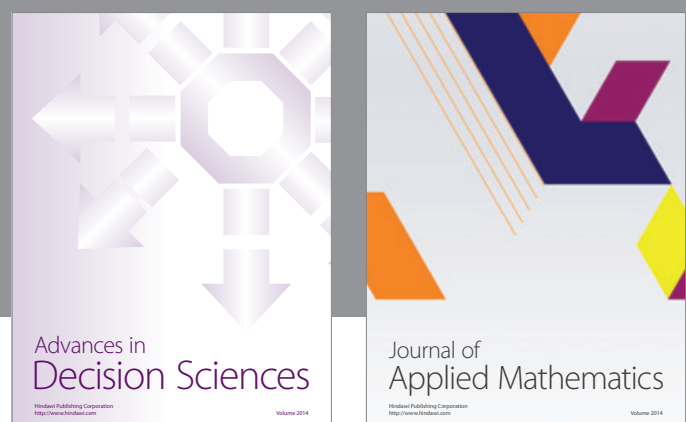

Journal of

Applied Mathematics
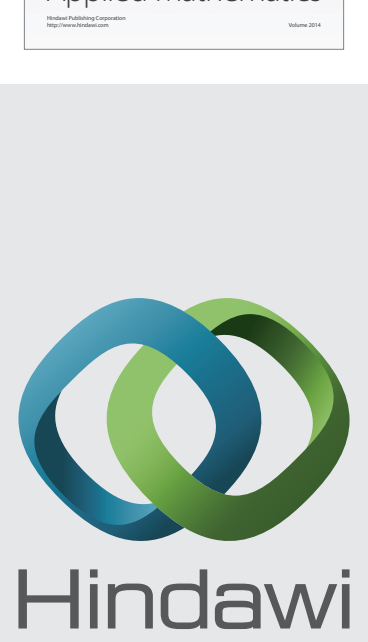

Submit your manuscripts at http://www.hindawi.com
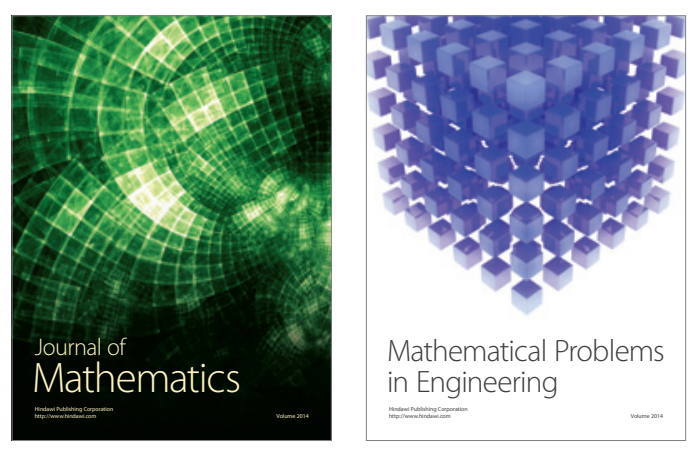

Mathematical Problems in Engineering
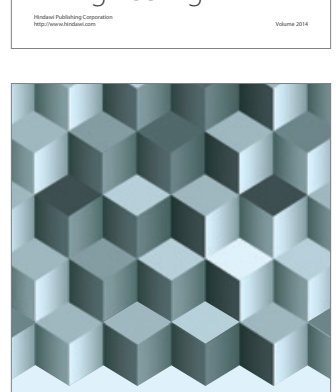

Journal of

Function Spaces
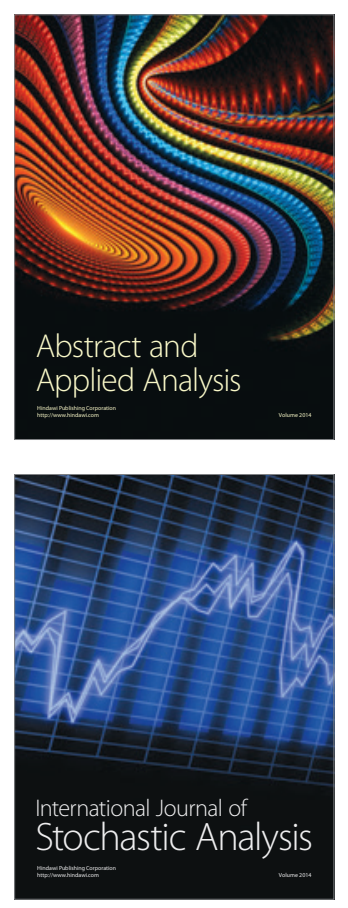

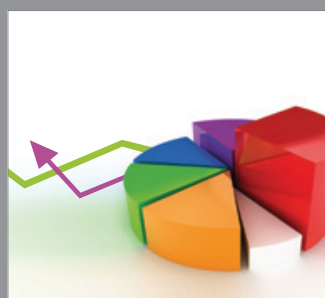

ournal of

Probability and Statistics

Promensencen
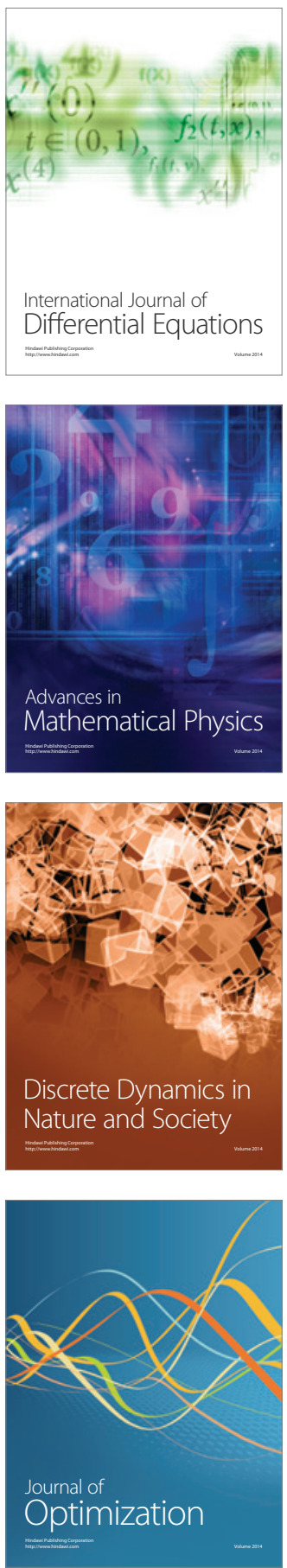